PREHOSPITAL CARE

\title{
Ambulance emergency services for patients with coronary heart disease in Lancashire: achieving standards and improving performance
}

\author{
B Stoykova, R Dowie, P Bastow, K V Rowsell, R P F Gregory
}

Emerg Med J 2004;21:99-104

See end of article for authors' affiliations

.....................

Correspondence to: Mrs B Stoykova, Health Economics Research Group, Brunel University, Uxbridge UB8 3PH, UK; Boyka.Stoykova@ brunel.ac.uk

Accepted for publication 17 December 2002

\begin{abstract}
Objectives: To examine the performance of a rural ambulance trust during two time periods, 1996/97 and 2001, with respect to achieving standards for ambulance journey times and delivery of clinical care for patients with suspected acute myocardial infarction (AMI).

Methods: Audit datasets on two cohorts of patients with chest pain and suspected AMI were assembled by the Lancashire Ambulance Service NHS Trust in north west England: 3706 patients during 1996/97 and 3423 in 2001 . They were transported to four hospitals. The analyses covered journey timings, role of rapid response vehicles (RRV), and clinical procedures and the results were compared with prevailing national standards.

Results: Hourly and daily usage patterns were similar in the two periods. During 1996/97 the national rural target of $95 \%$ of response times being within 19 minutes was achieved ( $96 \%$ of calls), unlike the target of 50\% within eight minutes (45.3\% of calls). During 2001, 2684 (78.4\%) calls had response times within eight minutes thus exceeding the revised national target of $75 \%$. RRVs were despatched for 1214 $(35.5 \%)$ of calls in 2001 , and the mean response time (SD) for these vehicles was significantly shorter than for front line ambulances (0:05:53 (0:02:49) versus 0:07:04 (0:04:19), $\mathrm{p}<0.001)$, likewise the mean call to hospital time (0:32:38 (0:09:28) v 0:35:01 (0:12:09), $\mathrm{p}<0.001)$. Patients in 2001 were more likely to be given aspirin by the ambulance crews (74\% of cases), while the rate of cannulation was lower.

Conclusion: A significant improvement has been achieved in the performance of ambulance services in Lancashire since 1996, because of recently introduced strategies, notably RRVs, and in the presence of more demanding national standards and targets.
\end{abstract}

$\mathrm{T}$ he national service framework (NSF) for coronary heart disease, published by the Department of Health in March $2000,{ }^{1}$ covered the role of ambulance service NHS trusts in dealing with patients with suspected acute myocardial infarction (AMI) and set out organisational goals and milestones. Ambulance performance standards for all emergency calls had first been set at a national level in 1974 and they were revised in $1996 .^{2}$ The standards were applicable to over 30 ambulance trusts in England, with trusts being classified as "urban services" if the population density of the geographical area that they covered was greater than 2.5 persons per square acre in 1991 or "rural services" if the density was lower. ${ }^{3}$

A number of strategies have been adopted by ambulance trusts to meet the cardiac standards. Paramedic crew members and defibrillator units are now available on front line ambulances, community first responder schemes have been introduced, and service coverage has been improved with the introduction of "rapid response" vehicles (RRV) (one person motorcycle or vehicle). But an assessment of the impact of such strategies on the performance of an ambulance trust has not been available. This paper examines the performance of the Lancashire Ambulance Service NHS Trust (LAS), a rural service in north west England, during two time periods, 1996/97 and 2001, with respect to achieving standards of timeliness and delivery of clinical care for patients with suspected AMI.

\section{METHODS}

Identification of standards

In England in 1996 calls for ambulances were categorised as "emergency" or "urgent". All 999 calls were classified as emergency calls. Urgent calls were made only by general practitioners (GP) and ambulance services were expected to respond within one or two hours. Prioritisation of emergency calls as category A or category B was introduced from 1997 onwards ${ }^{4}$ to ensure that immediately life threatening cases would get the fastest response. LAS introduced the system in October 1997. By 2001 the call prioritisation system required that ambulance calls were assessed as category A if the circumstances were immediately life threatening, as category $\mathrm{B}$ for other emergency calls, and as category $\mathrm{C}$ if the circumstances were not life threatening or serious. The prioritisation was done by ambulance despatch personnel according to the information provided by callers. Targets for response times according to category of call were set nationally. Targets for 1996 and 2001 are shown in table 1.

During the 1990s performance standards for calls to patients with suspected AMI were identified locally in contracts for clinical audit between ambulance trusts and health authorities purchasing their services. The standards in the 1997 contract between the North West Lancashire Health Authority and LAS included taking basic observations, recording a 3 lead electrocardiogram (ECG), administering aspirin as appropriate, and intravenous (IV) cannulation ${ }^{5}$ (table 1).

During 2001 standards were redefined nationally in accordance with the NSF recommendations. All calls for

Abbreviations: $A M I$, acute myocardial infarction; RRV, rapid response vehicle; NSF, national service framework; IV, intravenous; ECG, electrodardiogram; BVH, Blackpool Victoria Hospital 
Table 1 Performance standards valid during the two periods of data collection

\begin{tabular}{|c|c|c|c|c|c|}
\hline & \multirow[b]{2}{*}{ Category } & \multicolumn{2}{|l|}{ Period A 1996/97 } & \multicolumn{2}{|l|}{ Period B 2001} \\
\hline & & Standards and targets & $\begin{array}{l}\text { Source } \\
\text { (reference) }\end{array}$ & Standards and targets & $\begin{array}{l}\text { Source } \\
\text { (reference) }\end{array}$ \\
\hline \multirow[t]{4}{*}{ General } & Types of calls & $\begin{array}{l}\text { Emergency calls } \\
\text { Doctors' urgent calls }\end{array}$ & 3 & $\begin{array}{l}\text { Category } \mathrm{A}, \mathrm{B} \text { and } \mathrm{C} \text { calls } \\
\text { Doctors' urgent calls }^{\prime}\end{array}$ & 2,3 \\
\hline & $\begin{array}{l}\text { Response time for all emergency } \\
\text { calls (call to scene time) }\end{array}$ & $8 \mathrm{~min}$ for $50 \%$ of calls (all areas) & 3 & $\begin{array}{l}\text { Cat. A: } 8 \text { min } 0 \mathrm{sec} \text { for } \\
75 \% \text { of calls (all areas) }\end{array}$ & 2,3 \\
\hline & & 14 min for $95 \%$ of calls (urban areas) & & $\begin{array}{l}\text { Cat. B: } 14 \text { min } 0 \text { sec for } \\
95 \% \text { of calls (urban) }\end{array}$ & \\
\hline & & 19 min for $95 \%$ of calls (rural areas) & & $\begin{array}{l}19 \text { min } 0 \text { sec for } 95 \% \\
\text { of calls (rural) }\end{array}$ & \\
\hline \multirow[t]{11}{*}{$\begin{array}{l}\text { If } A M I \text { is } \\
\text { suspected }\end{array}$} & Time at scene & $\begin{array}{l}15 \mathrm{~min} \text { ( } 80 \% \text { for non-cardiac arrest } \\
\text { cases) }\end{array}$ & 5 & Not explicitly specified & - \\
\hline & Time to reach hospital & 10 min for $80 \%$ of calls & 5 & Not explicitly specified & - \\
\hline & Call to hospital time & Not explicitly specified & 5 & $\begin{array}{l}30 \text { min } 0 \text { sec for patients eligible } \\
\text { for thrombolysis (\% not specified) }\end{array}$ & 1 \\
\hline & "First responder" scheme & Not applicable. & - & Applicable. & 1 \\
\hline & Delivery of clinical care & Locally developed protocols & 5 & An agreed service- wide protocol & 1 \\
\hline & - ECG & 3 lead (in $90 \%$ of cases) & 5 & 12 lead (if available) & 1 \\
\hline & - Aspirin & $\begin{array}{l}\text { Yes, if there are no contraindications } \\
\text { including aspirin already given }\end{array}$ & 5 & Yes, at least $300 \mathrm{mg}$ orally & 1 \\
\hline & - IV access & $\begin{array}{l}\text { Yes: refused in less than } 5 \% \text {; successful } \\
\text { in } 90 \% \text { of the remainder. }\end{array}$ & 5 & $\begin{array}{l}\text { Not explicitly specified in the } \\
\text { framework, but recommended in } \\
\text { the guidelines }\end{array}$ & 1,6 \\
\hline & $\begin{array}{l}\text { - Pain relief and high } \\
\text { concentration oxygen }\end{array}$ & Not explicitly specified & 5 & Yes & 1 \\
\hline & - Defibrillator on scene & Yes & 5 & Yes & 1 \\
\hline & - Prehospital thrombolysis & Not applicable. & - & Yes, if call to hospital time is $>30 \mathrm{~min}$ & 1 \\
\hline
\end{tabular}

suspected AMI were to be classified as category A. Patients should receive, unless contraindicated, cardiopulmonary resuscitation and defibrillation in the event of cardiac arrests, high concentration oxygen, pain relief, aspirin (at least $300 \mathrm{mg}$ orally), and immediate transfer to hospital. The Joint Royal Colleges Ambulance Service Liaison Committee developed further detailed guidelines on the prehospital care, which patients with suspected cardiac chest pain should receive. ${ }^{6}$

\section{Setting}

LAS provides community emergency services for the county of Lancashire, which has a population of 1.4 million people and an area of 1100 square miles. ${ }^{7}$ There are 24 ambulance stations strategically located across the county. Forty three emergency ambulances and 10 RRVs (one motorcycle and nine cars), all equipped with a paramedic and defibrillator, are used at peak times. Since 1999 community first responder schemes have been operating in the Blackpool area. They include GPs and trained members of the public. ${ }^{8}$

\section{Data collection}

Patients with chest pain and symptoms indicative of ischaemic heart disease and possible AMI, who were attended by an ambulance crew, were eligible for the study. Data were collected over two periods of time: between 1 August 1996 and 31 October 1997 (group A) and from 1 January to 31 December 2001 (group B). The 1996/97 data collection exercise was an audit for a research project. ${ }^{9}{ }^{10}$ The audit was undertaken in 12 stations covered by the North West and South Lancashire Health Authorities. Patients were transported mainly to four district hospitals: Blackpool Victoria Hospital (BVH), Chorley District Hospital, Ormskirk General Hospital, and Royal Preston Hospital. The group A data were extracted from patient report forms by ambulance personnel and entered onto a computer program written in Visual Basic 3.0. The 2001 data collection exercise was a routinely conducted audit recommended by the NSF for coronary heart disease. ${ }^{1}$ This exercise covered all eligible patients transported to BVH only. Data were collected for five ambulance stations in the Blackpool area. During this period three RRVs were based at the Blackpool station seven days a week. The motorcycle was used between 09.00 and 17.00, weather permitting, while the cars were available between 08.00 and 24.00. The group B data were extracted from patient report forms by PB and entered on a Microsoft Excel spreadsheet.

The following items were recorded over both observation periods: age and sex of patients; person who called for help (GP or other caller); category of call; ambulance timings (time of call, response time, time at scene, and time to reach hospital); and procedures carried out by the ambulance crew. During the second observation period the involvement of a RRV or a community responder was also recorded.

The two observation periods differed in the methods of recording time intervals. During 1996/97 all times were recorded to the nearest minute only, unlike in 2001 when time was recorded in minutes and seconds. Also, before April 1998 response time was defined as starting from receipt of the emergency call, while in 2001 it started after the address had been taken.

\section{Analyses}

Analyses were conducted at two levels. The first level applied to the full datasets for the two observation periods (group A for 1996/97 and group B for 2001). These analyses focused on ambulance usage patterns and journey timings. To minimise possible biases, second level analyses on journey timings and clinical procedures were carried out on two subgroups within group A and group B. Patients with cardiac arrest, both witnessed and unwitnessed, were excluded from the analyses.

The two datasets were analysed using a statistical software package (SPSS 9.0). Results are reported in descriptive statistics, or as mean, standard deviation (SD) and median. Analysis of variance $(F)$ and $\chi^{2}$ significance tests were applied where appropriate. As the response time measurements were not strictly comparable across the two periods, the analyses exclude comparative assessments of mean and median response rates. 


\section{RESULTS}

\section{Baseline characteristics of group A and group B}

Over the first observation period, 1996/97, 3706 journeys were recorded in 12 ambulance stations for patients with eligible chest pain symptoms. A total of 3337 calls (90\%) were emergency 999 calls and 369 (10\%) were calls classified by GPs as urgent: $311(8.4 \%)$ were urgent within one hour and $58(1.6 \%)$ were urgent within two hours. During the second observation period, 2001, 3423 eligible patients were transported by ambulance to BVH. All calls were classified as category A.

\section{Patterns in the ambulance workloads}

There was little variation in the patterns of the ambulance workloads during the two study periods. The mean (SD) numbers of calls per month were 261.1 (56.8) calls during $1996 / 97$ and 285.3 (62.7) calls in 2001. The daily call patterns were similar with Mondays receiving 15\% and Sundays 14\% of calls in both periods. The hourly workloads, likewise, were similar: in both periods the demand for ambulance services was at its peak between 08.00 and 13.00. GPs placed significantly fewer calls in the more recent period, $18.0 \%$ (616) compared with $31.0 \%$ (1149) in 1996/97 ( $<<0.001$, $\left.\chi^{2}=160.77\right)$. A small number of the 2001 calls were via NHS Direct $(107,3.1 \%)$.

\section{Comparative response times}

During 1996/97, 3201 (96\%) of all 3337 emergency calls had response times within 19 minutes thus achieving the $95 \%$ national target for rural areas shown in table 1 . The response time target of eight minutes for $50 \%$ of calls was nearly achieved as there were 1510 calls $(45.3 \%)$ within eight minutes. During 2001, using the more precise timing practice, $2684(78.4 \%)$ calls had response times within the current target of 8 minutes 0 seconds thus exceeding the national target of $75 \%$.

A community first responder or a RRV arrived at the scene for $1377(40.2 \%)$ of all calls in 2001 ; for $1214(88.2 \%)$ of these calls it was a RRV. Table 2 shows that the RRV calls had a significantly shorter mean response $(\mathrm{p}<0.001)$. As a result, the response time of calls with a dispatched RRV was within the 8 min $0 \mathrm{~s}$ target for $88.2 \%$ of the calls, compared with $72.9 \%$ achieved by the remainder of the calls $(p<0.001$, $\left.\chi^{2}=108.01\right)$. Both the mean and median time at scene recorded for RRV patients was a minute longer $(\mathrm{p}<0.001)$ (see table 2), partly because the time at scene measurement included the interval between the arrival of the RRV and the ambulance following behind. Nevertheless, the overall call to hospital time was significantly shorter for the RRV cases $(\mathrm{p}<0.001)$.

\section{Subgroup comparison}

To assess the management of the patients against the prevailing standards for suspected AMI, analyses were carried out at a subgroup level. The subgroups for 1996/97 and 2001 were patients transported to BVH who were treated by ambulance crews according to LAS protocols for chest pain of cardiac origin and suspected AMI. Subgroup A (1996/97) had 2247 patients and subgroup B (2001) had 1234 patients. The two subgroups were similar in terms of the ratio between men and women and their average ages (table 3 ).

The national standard in 2001 for the total time from call to reaching hospital for patients eligible for thrombolysis was 30 minutes 0 seconds (table 1 ). For all patients in subgroup $\mathrm{A}$ in 1996/97, this time interval was achieved for $24.6 \%$ (552) of calls; the rate five years later for all subgroup B patients was significantly higher at $38.6 \%$ (476 calls) ( $p<0.001)$-see table 3.

Mean time at scene was the same for the two subgroups whereas mean journey time to hospital was shorter in 2001 by two minutes $(\mathrm{p}<0.001)$. Time reductions also occurred during the response phase as $79 \%$ of subgroup B patients were reached within 8 minutes 0 seconds.

Table 3 compares the rates for procedures and medications given to the subgroup patients in the two time periods. Aspirin was given to $74 \%$ of subgroup B patients compared with the earlier rate of $55 \%$ for subgroup A patients, although $15 \%$ in this earlier period had contraindications. The rates for IV cannulation were the reverse of the aspirin rates. Administration of high concentration oxygen was audited in 2001 and it was given to $89 \%$ of subgroup B patients.

\section{Effect of RRVs on times and procedures}

As 39\% (483) of subgroup B patients were attended by rapid response paramedics, we assessed their impact. As expected from the analysis of group B patients for 2001 in table 2, there was a significant reduction in response time and call to hospital time when a RRV was despatched to the scene of a suspected AMI (table 4). No statistically significant differences were found however in the comparative rates for clinical procedures performed by ambulance crews who were first on scene and crews who followed a rapid response paramedic.

Table 2 Time intervals for rapid response vehicles and others: group B (2001)

\begin{tabular}{|c|c|c|c|c|}
\hline & & \multicolumn{2}{|c|}{ Rapid response vehicle dispatched } & \multirow[b]{2}{*}{ p Value } \\
\hline & & Yes & No & \\
\hline Response time & $\begin{array}{l}\text { Mean } \\
\text { (SD) } \\
\text { Median } \\
\mathrm{N}\end{array}$ & $\begin{array}{l}0: 05: 53 \\
(0: 02: 49) \\
0: 05: 31 \\
1214\end{array}$ & $\begin{array}{l}0: 07: 04 \\
(0: 04: 19) \\
0: 06: 06 \\
2209\end{array}$ & $\mathrm{p}<0.001, F=74.99$ \\
\hline Time at scene & $\begin{array}{l}\text { Mean } \\
\text { (SD) } \\
\text { Median } \\
\mathrm{N}\end{array}$ & $\begin{array}{l}0: 16: 30 \\
(0: 06: 40) \\
0: 15: 40 \\
1214\end{array}$ & $\begin{array}{l}0: 15: 35 \\
(0: 07: 02) \\
0: 14: 39 \\
2209\end{array}$ & $\mathrm{p}<0.001, F=14.00$ \\
\hline $\begin{array}{l}\text { Time to reach } \\
\text { hospital }\end{array}$ & $\begin{array}{l}\text { Mean } \\
\text { (SD) } \\
\text { Median } \\
\mathrm{N}\end{array}$ & $\begin{array}{l}0: 10: 14 \\
(0: 05: 29) \\
0: 08: 44 \\
1214\end{array}$ & $\begin{array}{l}0: 12: 21 \\
(0: 07: 04) \\
0: 10: 42 \\
2206\end{array}$ & $p<0.001, F=81.48$ \\
\hline Call to hospital time & $\begin{array}{l}\text { Mean } \\
\text { (SD) } \\
\text { Median } \\
\mathrm{N}\end{array}$ & $\begin{array}{l}0: 32: 38 \\
(0: 09: 28) \\
0: 30: 56 \\
1214\end{array}$ & $\begin{array}{l}0: 35: 01 \\
(0: 12: 09) \\
0: 33: 15 \\
2206\end{array}$ & $\mathrm{p}<0.001, F=34.68$ \\
\hline
\end{tabular}


Table 3 Comparison of baseline characteristics, times and procedures at subgroup level

\begin{tabular}{|c|c|c|c|c|}
\hline & & $\begin{array}{l}\text { Subgroup A } \\
1996 / 97 \\
(n=2247)\end{array}$ & $\begin{array}{l}\text { Subgroup B } \\
2001 \\
(n=1234)\end{array}$ & p Value \\
\hline Sex & Female & $913(40.6 \%)$ & $527(42.7 \%)$ & $p=0.234, \chi^{2}=1.41$ \\
\hline$(n, \%)$ & Male & $1334(59.4 \%)$ & $706(57.3 \%)$ & \\
\hline Age & Female & $71.45(13.20)$ & $69.52(14.31)$ & $p=0.010, F=6.70$ \\
\hline (mean, SD) & Male & 65.83 (13.29) & $64.06(15.17)$ & $p=0.007, F=7.42$ \\
\hline $\begin{array}{l}\text { Call to hospital } \\
\text { target } 2001\end{array}$ & $<30$ min & $552(24.6 \%)$ & $476(38.6 \%)$ & $\mathrm{p}<0.001, \chi^{2}=74.82$ \\
\hline $\begin{array}{l}\text { Response time } \\
\text { target }\end{array}$ & $<8 \min ^{*}$ & $1291(57.5 \%)$ & $975(79 \%)$ & $p<0.001, \chi^{2}=162.17$ \\
\hline \multirow[t]{3}{*}{ Time at scene } & Mean & $0: 17: 02$ & $0: 17: 16$ & $p=0.297, F=1.09$ \\
\hline & (SD) & (0:06:16) & $(0: 06: 20)$ & \\
\hline & Median & $0: 16: 00$ & $0: 16: 29$ & \\
\hline \multirow{3}{*}{$\begin{array}{l}\text { Time to reach } \\
\text { hospital }\end{array}$} & Mean & $0: 12: 02$ & $0: 10: 32$ & $p<0.001, F=48.18$ \\
\hline & (SD) & (0:06:13) & $(0: 05: 51)$ & \\
\hline & Median & $0: 11: 00$ & $0: 09: 00$ & \\
\hline \multirow{3}{*}{$\begin{array}{l}\text { Call to } \\
\text { hospital time }\end{array}$} & Mean & $0: 40: 22$ & $0: 34: 31$ & $p<0.001, F=126.51$ \\
\hline & (SD) & (0:16:30) & (0:10:29) & \\
\hline & Median & $0: 37: 00$ & $0: 32: 48$ & \\
\hline \multirow[t]{3}{*}{ Aspirin } & Given & 1227 (54.6\%) & $910(73.7 \%)$ & $p<0.001, \chi^{2}=196.38$ \\
\hline & Refused & $0(0 \%)$ & $9(0.7 \%)$ & \\
\hline & Contraindicated & $337(15.0 \%)$ & $30(2.4 \%)$ & \\
\hline ECG & & $2108(93.8 \%)$ & $1234(100 \%)$ & $p<0.001, \chi^{2}=165.92$ \\
\hline Cannulation & & 1670 (74.3\%) & $676(54.8 \%)$ & $\mathrm{p}<0.001, \chi^{2}=138.41$ \\
\hline Pain relief & & $243(10.8 \%)$ & $155(12.6 \%)$ & $p=0.096, \chi^{2}=2.77$ \\
\hline Oxygen & & No data & $1103(89.4 \%)$ & - \\
\hline
\end{tabular}

\section{DISCUSSION}

\section{Strengths and weaknesses of the study}

This study represents an opportunity to examine changes in the performance of ambulance services in Lancashire for patients with symptoms indicative of ischaemic heart disease. Although the two datasets differed in geographical coverage and timescales for data collection, by undertaking subgroup analyses for patients managed according to AMI protocols we were able to deal with these differences and minimise possible biases. Policies for recording journey time changed between the study periods. In 2001 the clock was started later for measuring response times (that is, after the address was ascertained), while precision was improved by recording both minutes and seconds. These changed practices meant that strict comparisons between response rates could not be made. Nevertheless, the results for each time period, 1996/7 or 2001, were robust and they could be compared with the appropriate targets. Both datasets were compiled prospectively, which contributed to their quality and completeness.

\section{Progress towards meeting current standards}

Although the time elapsed between the two study periods was comparatively short, in the intervening period there were important developments in the provision and performance of community emergency services. National standards were redefined based on NHS priorities and on existing research evidence and they posed new challenges to ambulance trusts over providing faster services and delivering optimal clinical care.

We found that over five years the rate achieved by LAS for meeting the eight minutes response time target rose from less than half to four fifths of calls. Improvements in performance were partly attributable to the new arrangements for deploying RRVs. For instance, in the Blackpool area in 2001, RRVs conformed to the eight minute response time

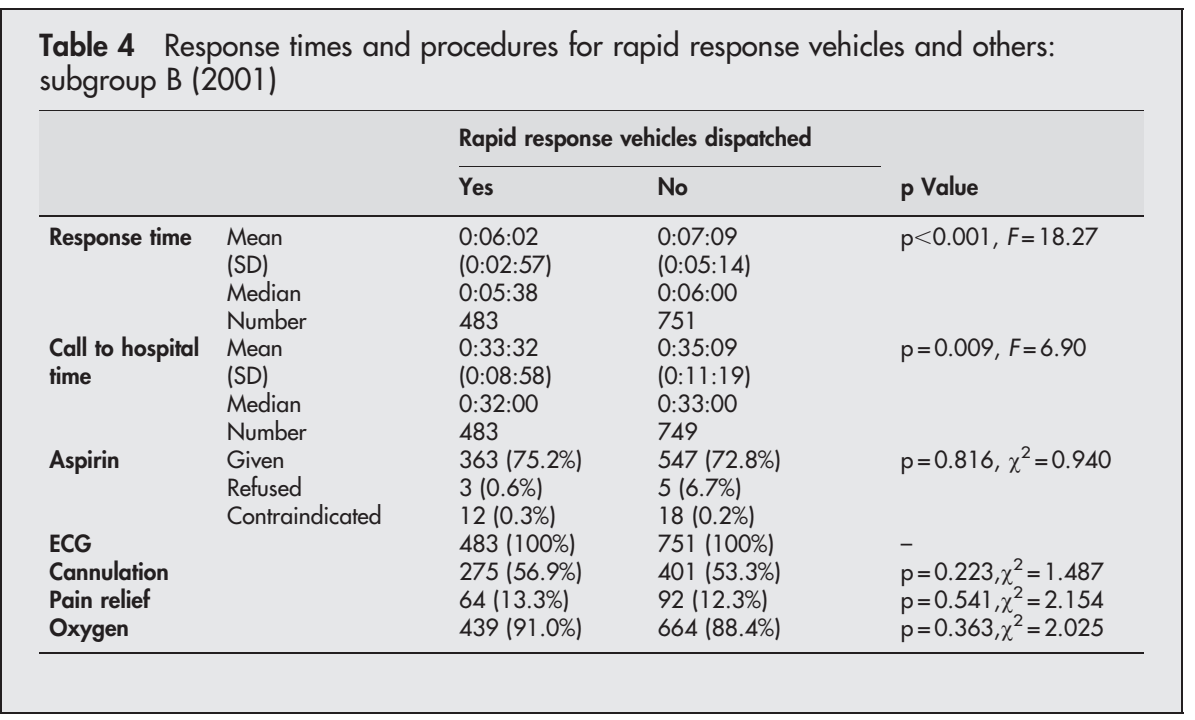


standard on almost $90 \%$ of occasions. This finding suggests that RRV schemes are a powerful means for achieving standards set by the NSF for coronary heart disease.

Hassan and Barnett ${ }^{11}$ recently reported that there was a consensus among a group of experts from ambulance trusts in the United Kingdom that a first responder system should be trained and fully integrated into an emergency medical service system. In addition, RRVs have been introduced in an increasing number of ambulance services in the past decade, but their effect on quality improvement of the services has not yet been studied in sufficient detail. The current Department of Heath routine data collection form (form KA34) does not require separate entries for calls attended by RRVs or approved first responders, thus relevant statistics are not reported in the annual national statistics bulletin. ${ }^{12}$ If further evidence was made available it could support decisions made locally by NHS ambulance trusts with regard to purchasing new vehicles as well as developing local first responder schemes. In Lancashire funding for these services has been provided by charities (by the "Heart of Lancashire" Appeal and other locally raised funds). ${ }^{8}$ Elsewhere, similar service innovation may carry a significant budgetary impact if unsupported by charitable funds.

The NSF 30 minute target from the time of call until reaching hospital seemed to be a challenge during 2001. Although we observed shorter call to hospital times in 2001 compared with 1996/97, they still exceeded the target (the median was nearly 33 minutes). As the framework's standard for call to hospital time was intended for patients eligible for thrombolysis, our results really cannot be judged against the target because assessments of patients' suitability for thrombolysis were not recorded. Indeed, it would be difficult for ambulance services to collect information about eligibility for thrombolytic treatment, as assessments need to be made retrospectively after confirmation of the diagnosis. The framework should provide instructions on how these data should best be collected and reported.

Although studies reporting response times and other time intervals achieved by comparable ambulance services in the British Isles have been published, they have been concerned primarily with the overall performance of a service over a single time period, ${ }^{13}$ specific time intervals, such as call to needle time ${ }^{14}$ and time on scene, ${ }^{15}$ or have focused on out of hospital cardiac arrests. ${ }^{16}{ }^{17}$ We have not found other time series data on ambulance performances with which to compare the performances of LAS.

\section{Standards for treating suspected AMI}

This study identified some differences between the clinical care delivered in 1996/97 and 2001. The clinical guidelines used in LAS during both periods, ${ }^{56}$ required obtaining IV access if AMI was suspected. Still the proportions of cannulated patients decreased significantly between 1996/ 97 and 2001. A possible explanation of this observation could be the improved training of paramedics, so that they are now able to exercise judgement over appropriate treatments rather than systematically following instructions from clinical protocols.

Patients were more likely to be given aspirin in 2001 because, according to the prevailing LAS protocol, only major contraindications were to be taken into consideration. The optimal level in 2001 was 300 mg. Patients who had already taken smaller amounts of aspirin were given a supplement and this practice also contributed to the higher recorded rate for aspirin administration in 2001. Woollard and colleagues surveyed protocols on aspirin use followed by nine ambulance services in England and Wales and found wide variation in the identification of contraindications. They concluded that a standardised protocol should be devised that minimised the number of contraindications. ${ }^{18}$

\section{Future improvements for ambulance services}

The NSF for coronary heart disease suggested that prehospital thrombolysis for patients with AMI should be considered under certain circumstances, but the policy would have resource implications for ambulance trusts. LAS is considering prehospital thrombolysis and has provided additional training for paramedics and begun installing 12 lead ECG machines and telemetry on front line ambulances for establishing contact and obtaining advice from a hospital cardiologist. ${ }^{19}$ In Lancashire an electronic patient report system is being introduced, whereby LAS ambulance crews enter the patients' clinical details on handheld personal computers and the details are transferred electronically to the receiving hospital department. Giovas and colleagues demonstrated the benefits of shortening the time to reach an AMI diagnosis and starting thrombolysis when ECGs are transmitted from a moving ambulance to a hospital based cardiologist. ${ }^{20}$ The LAS electronic patient record system will also facilitate more comprehensive, faster and easier audits in accordance with another requirement of the NSF that ambulance trusts should undertake routine audits. ${ }^{1}$

In May 2002 the Department of Health in England announced an investment of $£ 14$ million to provide ambulances with 12 lead ECG machines and to enable the introduction of communications equipment for transmitting information while on route to hospital. ${ }^{21}$ The benefits of this investment should be studied. It would be useful also to undertake a comparative analysis of rural and urban ambulance services to establish if any amendments in current standards are appropriate, especially with respect to call to hospital times. But for the benefits of improved ambulance services in the provision of AMI care to be fully appreciated, a comprehensive evaluation is needed covering appropriate follow up periods and measuring health outcomes in the treated populations.

\section{ACKNOWLEDGEMENTS}

We wish to thank members of staff at the Lancashire Ambulance Service NHS Trust for their assistance in the two data collection periods and the North West Research and Development Directorate for funding the 1996/97 cardiac research project. We are grateful to Dr C J Harrison, Director of Public Health in the South Lancashire Health Authority at the time of setting up the 1996 survey, for help and encouragement. The Health Economics Research Group at Brunel University receives funding from the Department of Health Policy Research Programme.

\section{Authors' affiliations \\ B Stoykova, R Dowie, Health Economics Research Group, Brunel University, Uxbridge, UK \\ P Bastow, Lancashire Ambulance Service NHS Trust, Preston, UK \\ K V Rowsell, University of Central Lancashire, Preston, UK \\ R P F Gregory, University of Manchester, Manchester, UK}

\section{REFERENCES}

1 Department of Health. National service framework for coronary heart disease: modern standards and service models. London: Department of Health, 2000.

2 Department of Health. Modernisation of ambulance services. Health service circular 1999/091. http://www:tap.ccta.gov.uk/doh/coin4.nsf/ laccessed 30 Apr 2002).

3 Department of Health. Ambulance services England 2000-01. http:// www.doh.gov.uk/public/sb0115.htm (accessed 30 Apr 2002).

4 NHS Executive. Review of ambulance performance standards: final report of steering group. London: NHS Executive, 1996.

5 Lancashire Ambulance Service NHS Trust. Clinical Audit Report 1996/97. Lancaster: LAS NHS Trust, 1997.

6 Joint Royal Colleges Ambulance Service Liaison Committee. Pre-hospital guidelines 2002 for use in UK ambulance services [CD-ROM]. Version 2.0. Warwick: University of Warwick and QinetiQ, 2002. 
7 Economic Development Group. Area profiles. http:// www.lancashireprofile.com/ (accessed 13 May 2002).

8 Lancashire Ambulance service NHS trust. Annual Report and accounts 2001/ $2002 \mathrm{http}: / /$ www.lancashireambulance.com/downloads/ AnnualReport0102.pdf (accessed 13 Nov 2002).

9 Dowie R, Gregory RPF, Annis S, et al. Ambulance records-a mine of information. Clinician in Management 1997;6:2-4.

10 Dowie R, Gregory RP, Rowsell KV, et al. A decision analytic approach to commissioning ambulance cardiac services. Journal of Management and Medicine 1998;12:81-91.

11 Hassan TB, Barnett DB. Delphi type methodology to develop consensus on the future design of EMS systems in the United Kingdom. Emerg Med J 2002;19:155-9.

12 Department of Health. Ambulance services England 2001-02. http:// www.doh.gov.uk/public/sb0213/sb0213.pdf (accessed 18 Nov 2002).

13 Breen N, Woods J, Bury G, et al. A national census of ambulance response times to emergency calls in Ireland. J Accid Emerg Med 2000;17:392-5.

14 Rawles J, Sinclair $C$, Jennings $K$, et al. Call to needle time after acute myocardial infarction in urban and rural areas in northeast Scotland: prospective observational study. BMJ 1998;317:576-8.
15 Barrett B, Guly HR On scene time in incidents responded by urban and rural ambulances. Pre-hospital Immediate Care 1999;3:145-8.

16 Dowie R, Campbell H, Donohoe R, et al. "Event-tree" analysis of out-ofhospital cardiac arrest data: confirming the importance of bystander CPR. Resuscitation (in press)

17 Pell JP, Sirel JM, Marsden AK, et al. Effect of reducing ambulance response times on deaths from out of hospital cardiac arrest: cohort study. BMJ 2001;322:1385-8.

18 Woollard M, Smith A, Elwood P. Pre-hospital aspirin for suspected myocardial infarction and acute coronary syndromes: a headache for paramedics? Emerg Med J 2001;18:478-81.

19 Bastow P. Handheld PCs help ambulance staff deliver better patient care. NHS Information Authority News-March 2002. http:// www.lancashireambulance.com/downloads/inform 03021.pdf (accessed 30 Apr 2002).

20 Giovas P, Pappadoyannis D, Thomakos D, et al. Transmission of electrocardiograms from a moving ambulance. J Telemed Telecare 1998:4(suppl 1):5-7.

21 The National Health Service. NHS update. http://www.nhs.uk/nhsupdate/ news. asp?newsid $=435$ (accessed 5 Jun 2002). 